\title{
Automated sound recording and analysis techniques for bird surveys and conservation
}

\author{
T. SCOTT BRANDES
}

\begin{abstract}
Summary
There is a great need for increased use and further development of automated sound recording and analysis of avian sounds. Birds are critical to ecosystem functioning so techniques to make avian monitoring more efficient and accurate will greatly benefit science and conservation efforts. We provide an overview of the hardware approaches to automated sound recording as well as an overview of the prominent techniques used in software to automatically detect and classify avian sound. We provide a comparative summary of examples of three general categories of hardware solutions for automating sound recording which include a hardware interface for a scheduling timer to control a standalone commercial recorder, a programmable recording device, and a single board computer. We also describe examples of the two main approaches to improving microphone performance for automated recorders through small arrays of microphone elements and using waveguides. For the purposes of thinking about automated sound analysis, we suggest five basic sound fragment types of avian sound and discuss a variety of techniques to automatically detect and classify avian sounds to species level, as well as their limitations. A variety of the features to measure for the various call types are provided, along with a variety of classification methods for those features. They are discussed in context of general performance as well as the monitoring and conservation efforts they are used in.
\end{abstract}

\section{Introduction}

Acoustic communication in birds is rich and lends itself to one of the primary ways in which birds make their presence known to each other, as well as one of the most direct ways for humans to detect them, often at times when they are difficult to see. Further, the vast majority of birds have songs or calls that serve as a species-specific acoustic signature that readily announces their presence. This abundance of sound provides a readymade source of information that can be used to explore the composition of avian diversity in particular regions of interest.

There is a great need for avian monitoring in conservation efforts. Birds are the most speciose group of terrestrial vertebrates and they encompass a wider range of habitats than any other group of vertebrates on the planet. Birds are particularly abundant and diverse in the tropics, consisting of both generalists and specialists, as well as migrants and local breeders. They are important consumers at several trophic levels; they eat fruit, grains, nectar, insects, and vertebrates. As such wide-ranging consumers, birds contribute to a variety of important ecosystem functions. As insectivores, they play a role in controlling insect populations (Holmes 1990; Holmes et al. 1979), as frugivores they are particularly important plant dispersal agents (Snow 1971, 1981, Howe and Smallwood 1982), and as nectar consumers they are important pollinators (Carpenter 1978, Feinsinger and Colwell 1978, Proctor et al. 1996). Since birds play such varied and widespread roles in ecosystem functions, they are vulnerable to both human induced habitat change (Becker and Agreda 2005, Canterbury and Blockstein 1997) and global climate change (Pounds et al. 1999), and as a result many species are declining (Collar et al. 1994). 
Bird sound is the most efficient means for surveying birds, particularly in the tropics (Parker 1991, Riede 1993, Kroodsma et al. 1996). A variety of methods for monitoring and assessing birds are available (Ralph and Scott 1981, Verner 1985, Bibby et al. 1992, and Ralph et al. 1993) and three widely used techniques are mist-netting, point counts, and transect counts. Of these, only mist-netting does not include the use of passive acoustics. Point and transect counts that make use of bird sounds are more efficient than mist-nets (Parker 1991, Angehr et al. 2002).

Acoustic surveying lends itself to rapid assessment programmes (RAP) which quickly evaluate the biodiversity of specific regions (Riede 1998). This is largely because birds are heard more often than seen or trapped. This translates into not only more accurate species counts, but also faster estimations of biodiversity. Parker (1991) describes how in seven days he recorded the vocalizations of $85 \%$ of the 287 species of avifauna his team of seven ornithologists inventoried after 54 days of intensive field work within a $2 \mathrm{~km}^{2}$ area in Amazonian Bolivia, which included 36,804 mist-net hours.

The most significant drawback to point and transect counts is the reliance on highly trained personnel for making identifications of species and the inherent subjectivity of their data due to skill level. This can make comparisons between data from different personnel unreliable (Angehr et al. 2002). However, the use of acoustic recorders can greatly reduce this variability, and some studies have suggested that acoustic recordings alone are preferable to trained personnel without recorders, since recordings are more consistent and achievable (Haselmayer and Quinn 2000, Hobson et al. 2002, Rempel et al. 2005). Additional standardisation in data collection is possible by using automated recorders, particularly those that can record on a programmable schedule. Although it is often not possible to directly count the number of calling individuals this way, there are methods available for estimating associated information useful for conservation research. For instance, abundance estimates are needed to make use of many metrics of biodiversity change, and automated recorders scheduled to record at the same time and spaced far enough apart to not record the same individuals can be used to provide these estimates through occupancy modelling (Royle and Nichols 2003, Mackenzie et al. 2005).

Having this process automated can involve more than just having the recording itself automated; it also includes automating the sound analysis and species identification. This automation can take the form of simple template matching or more advanced techniques such as those used in human speech recognition. There is still much work to be done with automatic call recognition (ACR) software, particularly with classifying a wide range of bird species recorded in a natural setting and detecting novel calls, but promising inroads have been made and some ACR methods have been shown to work in suitable settings. The remainder of this work is organized as follows. A summary of useful hardware approaches to automating acoustic recording of bird sound is provided. Then, an overview of approaches to automated analysis of bird sound is given followed by concluding thoughts and direction of future research.

\section{Hardware for automated recording of bird sounds}

\section{Hardware for scheduled recordings}

The basic components of hardware for use in automated recording of bird sound are the microphone, audio recorder, power supply, mechanism for initiating and ending recordings, and a weather-proof housing for the equipment. The hardware described here consists of devices designed to operate autonomously in remote locations and record high quality digital sound stored on hard drives or flash memory cards at scheduled intervals of time. There are three primary approaches to developing this sort of recording apparatus. Here I provide details and examples of all three of these approaches. While the examples are by no means exhaustive, they are a representative overview of the three main categories of the current technology available, and a list of vendors is provided just before the reference section. The first but least sophisticated approach is to design a scheduling timer through a hardware interface to control a standalone commercial recorder. This is the least desirable option since it typically involves tampering with 
the recorder and using it in a way not intended by the engineers who designed it, but it is the least expensive. A commercially available example of this type of device is the programmable timer circuit board by Nauta, an Italian company, which is adapted to control the M-audio Microtrack 24/96 recorder. Though the field-ready product they sell is intended for cetaceans, they also sell their timer board by itself and provide detailed instructions for its installation and use, and it can be readily adapted for use in recording birds. This device saves sound as noncompressed wave-files. Devices that save sound as $\mathrm{mp}_{3}$ files or other compressed formats are less attractive since data is lost. This compression format is designed to be minimally detectable to human ears, so researchers should do a comparative analysis on their sounds recorded in both lossless and lossy sound recording formats to verify that their target sounds are not degraded by the recording format chosen, and not just listen to the recordings.

A more elegant approach is to write software for a programmable recording device, such as a personal digital assistant (PDA) or a smart phone. This approach is preferable to the previous one in that the recording device does not need to be tampered with and it lends itself to more complicated recording schedules. For instance, a recording schedule starting 30 minutes before sunrise, as opposed to a fixed clock time such as o6hoo, is more readily accomplished through software running on a PDA than with an external timer circuit board. A further benefit to these devices is that they are designed to be user-friendly and the scheduling software can be written for use by non-technical personnel. An example of this type of device is one built by Conservation International (Brandes 2005). In their device, an HP iPac 5550 is used to record sound at scheduled intervals and store it on 4 GB flash memory cards (this scheduling software is available at no cost from the Tropical Ecology Assessment and Monitoring Network at Conservation International or by sending an email request to the author). The PDA approach is not as power efficient as it could be, in that connecting it to an external battery supply begins the process of recharging the on-board Li-ion battery instead of powering the unit directly. Conservation International's PDA recorders require about 17 amp-hours of battery life to record to minutes of sound daily for two weeks, which can be accomplished with eight high-capacity NiMH batteries. Both of these approaches are susceptible to changes in hardware by the manufacturers in the form of firmware or operating system updates and much work might need to be done to keep up with the latest hardware available. This approach in particular has been used in monitoring nocturnal sounds in tropical forests, but it could readily be used to monitor diurnal avifauna.

The most sophisticated of the approaches presented here is to develop recorders with singleboard computers. This provides the developer with the most control in creating a device designed for the sole purpose of recording sound autonomously. To do this properly requires a dedicated engineering lab, and a few labs now offer their products commercially for purchase or for lease. An experienced lab in this area is the Bioacoustics Research Program (BRP) at the Cornel Lab of Ornithology. For many years they have been developing autonomous recording units (ARUs) for both terrestrial and underwater use. They currently offer these units for lease and can custom design the sampling schedule to fit particular requirements. They store sound onto an $80 \mathrm{~GB}$ hard drive and can be deployed for months at a time. BRP also makes ARUs that record in stereo and use a GPS time-stamp, enabling the recordings to be used to find the position of animals and track their movement. These recorders have been used for many years in the ongoing effort to find the Ivory-billed Woodpecker Campephilus principalus (Fitzpatrick et al. 2005), and this has contributed to their continued improvement. A private company, Wildlife Acoustics, now offers a single-board computer type of recorder commercially. It stores 8 o hours of sound and can be kept in the field for a month or longer on a single set of batteries. They have recently been used to monitor breeding Cerulean Warblers Dendroica cerulea by the U.S. Forest Service.

\section{Microphones for automated recording}

Microphones for hand-held field recordings can be quite specialized, and the same is true of those for automated recorders. Typically, microphones for handheld recordings are far more 
sensitive to sound in the direction they are pointed, preventing sounds incident at other angles from being recorded as loudly. This type of microphone works well when a researcher wants to record a particular individual that is singing. With automated recorders, the opposite is true, in that omni-directional microphones are used instead of directional microphones since there is no way of knowing ahead of time where a sound will originate. Single-element omni-directional microphones can be effective, but there are two basic approaches to increase their effectiveness. The first is to use a small array of microphone elements to create a more sensitive beam-pattern than that of a single microphone element. An example of this is the linear 16-element microphone array (less than $15 \mathrm{~cm}$ in length) designed by BRP for use with their ARUs. These arrays form a beam-pattern in the shape of a toroid (Figure $1, a$ ). They are most sensitive to sound around the axis of the microphone array, and least sensitive in the direction pointing from each end. By placing this microphone array in a canopy hanging downward, it is sensitive to sound originating from any direction within the canopy, and is least sensitive to sound originating directly above or below it. A second approach to improving omni-directional microphone gain is to use a specially designed waveguide to collect and amplify the sound before it reaches the microphone element (Figure 1, b). River Forks Research Corporation developed an omni-directional waveguide for the purpose of recording birds (Hobson et al. 2002). Their design consists of two $26.67 \mathrm{~cm}$ (10.5 inch) plastic discs stacked $2.54 \mathrm{~cm}$ (I inch) apart that have a hyperbolic curve in their inner surface that is used to amplify incoming sound. The microphone element is placed at the centre, between the discs. They are mounted horizontally, but are sensitive to sound above and below the waveguide as well as sound originating from all other angles. They are well suited to record birdsongs, but are more expensive than most high quality hand-held microphones.

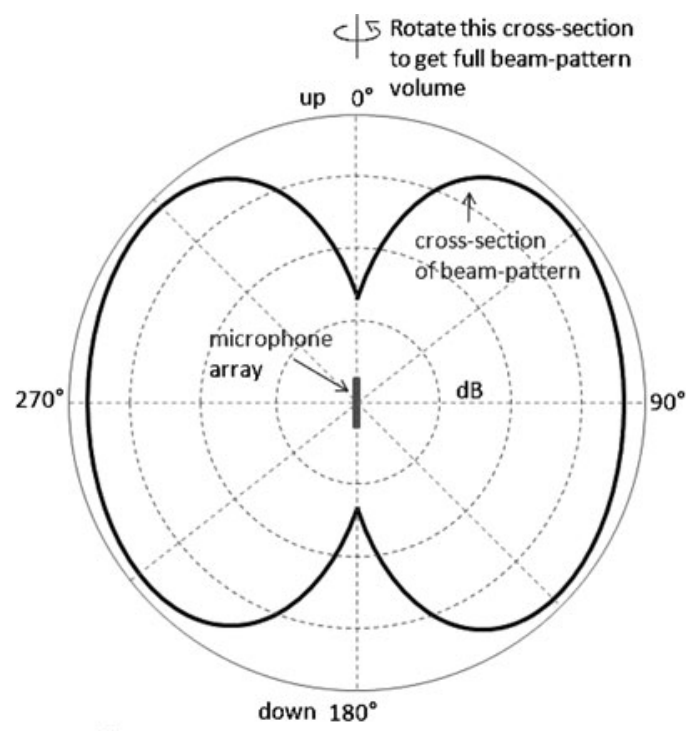

a.

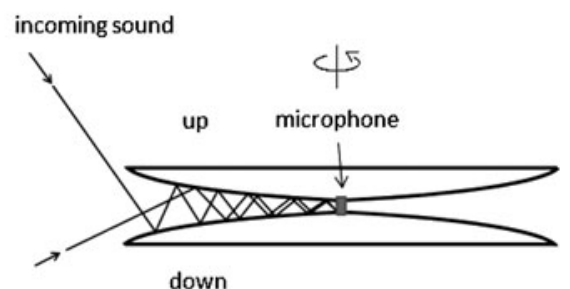

b.

Figure 1. (a.) Toroid shaped beam-pattern for a small microphone array and (b.) the hyperbolic waveguide. The beam-pattern indicates how sensitive the microphone is to sound as a function of angle. In both of these designs, sound originating from all around the microphone can be detected, but they are least sensitive to sound directly above or below. 


\section{Automated analysis of bird sounds}

Bird sounds as a whole are as complex and varied as any group of non-human animals. For the purposes of thinking about automated sound analysis, there are at least five broad categories of discrete sound unit shapes that compose bird sounds (Figure 2). These sound units include segments with constant frequency, frequency modulated whistles, broadband pulses, broadband with varying frequency components, and segments with strong harmonics. Even if this summarized all of the complexity of bird sounds, this degree of variety in sound types would be a challenging task for a single automated call recognition algorithm. Additionally, bird sounds often include complexity in the form of variations and themes of combinations of the basic sound shapes shown in Figure 2. If we think of those discrete chunks of sound as syllables, then this complexity can range from simple repeated sequences of syllables to complex sequences of syllables with patterns that seldom repeat. We can add to this complexity with field situations that make detection and classification more challenging when encountering duets, choruses of overlapping songs, intentional call masking, and vocal mimicry. Finally, difficulty in creating automated classifiers can arise from species that have regional dialects, very large song repertoires, and even improvisational songs.

At the least, automatic call recognition of bird songs is complex, but much has been done to make it viable when focused on a limited number of species. The analysis process has two primary parts, call feature extraction and call classification based on the features. The choice of which features to measure depends largely on the characteristic structure of the target calls, whereas the choice of classifier depends on the way in which the feature measurements distinguish the various types of target calls. A list of features and classifiers commonly used on a variety of call-types is provided in Table 1 .

Typically, features are chosen such that they provide information about a call that is characteristic about it, so that similar calls will be grouped together, and ones dissimilar will be recognized as being different. Features that consist of direct parameter measurements of a call, such as peak frequency and bandwidth, work well for constant frequency or simple frequency modulated calls. A variety of simple parameter measurements have been shown effective in nocturnal flight call monitoring of migrants (Farnsworth et al. 2004, Farnsworth and Russell, 2007, Hüppop et al. 2006). In particular, Schrama et al. (2008) chose seven direct measurements of calls to automate a process for recognizing nocturnal flight calls of migratory birds: call

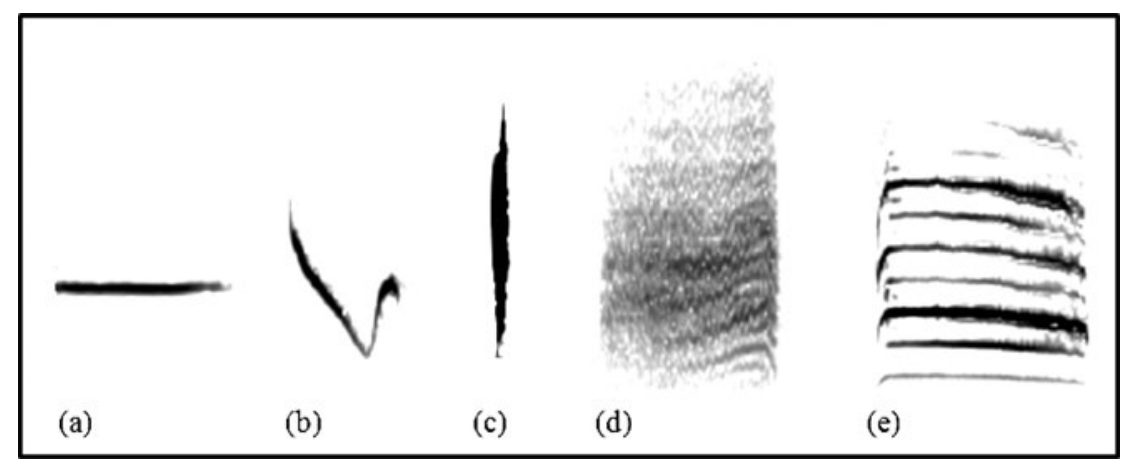

Figure 2. Spectrogram (frequency vs. time) displays of five broad categories of discrete sound unit shapes that compose bird sounds for the purpose of thinking about species-level automated sound recognition: (a) constant frequency (Wood Thrush Hylocichla mustelina); (b) frequency modulated whistles (Canon Wren Catherpes mexicanus); (c) broadband pulses (Brewer's Sparrow Spizella breweri); (d) broadband with varying frequency components (Canon Wren); (e) and segments with strong harmonics (Blue Jay Cyanocitta cristata). 
Table 1 . Comparison of the various features used to detect the basic sound unit shapes of bird sound in Figure 2, along with the various classification methods used with those features. This list serves as a summary of all the bird sound recognition methods referenced in this paper. Interestingly, none of the methods target call type ' $\mathrm{d}$ ', broadband with varying frequency components.

\begin{tabular}{|c|c|c|}
\hline Target Call Type in Figure 2 & Feature Extraction & Classification Method \\
\hline$a, b$ & $\begin{array}{l}\text { direct time and frequency } \\
\text { measures from target calls }\end{array}$ & $\begin{array}{l}\text { Bayesian classifier, Euclidian } \\
\text { distance }\end{array}$ \\
\hline c & pulse-to-pulse duration & neural networks \\
\hline c & sound template & $\begin{array}{l}\text { dynamic time warping, minimum } \\
\text { cross-correlation threshold }\end{array}$ \\
\hline e & $\begin{array}{l}\text { multi-spectral estimates with FFT } \\
\text { and related functions }\end{array}$ & multivariate statistics \\
\hline$a, b, e$ & peak frequency contour vector & $\begin{array}{l}\text { Bayesian classifier, dynamic time } \\
\text { warping, hidden Markov models, } \\
\text { neural networks }\end{array}$ \\
\hline $\mathrm{b}, \mathrm{e}$ & cepstral coefficients & $\begin{array}{l}\text { dynamic time warping, Gaussian } \\
\text { mixture models, hidden Markov } \\
\text { models }\end{array}$ \\
\hline
\end{tabular}

duration, highest frequency, lowest frequency, loudest frequency, average bandwidth, maximum bandwidth and average frequency slope. If the call type clusters resulting from features are linearly separable, then simple Bayesian classifiers can be used. Otherwise, neural networks can be employed to attempt to separate the call types. Terry and McGregor (2002) successfully used and compared three basic types of neural networks to identify individual Corncrakes Crex crex. Since Corncrakes utter calls that consist of broad-band pulses with distinct timing, they found that the pulse-to-pulse timing is the important feature to measure. In a novel approach, Tanttu et al. (2006) created their own type of frequency contour vector to use in self-organizing neural networks to recognize simple frequency modulated flight calls from crossbills Loxia spp. By focusing only on the peak harmonic in any multi-harmonic calls, they were able to extract the features needed to make correct identifications.

Another method that has been successful is template matching. In its basic form, this is done by selecting an example sound as a template with which to find other instances of this sound. This approach can work if the sound has little variation among individual birds and if the background sounds are similar in both the template and the field recordings. This is the approach used in the ongoing effort to find the Ivory-billed Woodpecker. Though template matching typically works better with longer signals, it is appropriate to try with Ivory-billed Woodpeckers since they have simple calls that have little variation and they also make distinct double rap sounds on trees. Additionally, there are not many sample recordings of them, so a method with an adjustable threshold for positive identification allows a wider variation of similar calls to be marked for more detailed analysis. A more sophisticated approach is to use dynamic time warping (DTW) in the template matching (Anderson et al. 1996). DTW is performed by expanding and contracting the template in time to better fit songs given a little slower or faster than the template sound. This allows for a wider range of detections than the standard template matching, but it only works on species which have relatively consistent songs or calls. Additionally, it requires a template for each sound to identify, so it works most efficiently with species that have very small and consistent repertoires. Schrama et al. (2008) have shown success using DTW to better detect nocturnal migrants by their flight call.

An entirely different approach is to use stochastic sequence modelling techniques to make sound classifications based on short-time measurements of sound features and how those features change in time. This is accomplished with hidden Markov models (HMM), a technique widely used for human speech recognition. The features most commonly used for human speech recognition are calculated with cepstral coefficients and derivatives thereof. These coefficients 
capture the rich harmonic structure of human voice well, and the sequences of their change throughout the course of speaking a word can be modelled accurately with HMMs. These methods have been successfully used to classify bird songs (Kogan and Margoliash 1998, Trifa et al. 2008), particularly with birds that have a rich harmonic structure in their songs. Cepstral coefficients have some drawbacks though. In particular, they are very susceptible to noise and can lead to poor classification of sounds that overlap in time, even if they do not overlap in frequency. Notably, Trifa et al. (2008) had success using HMMs to recognize antbirds (Thamnophilidae) in a Mexican rainforest. The species they chose do not have calls with strong harmonics and their recordings have some degree of background noise. Another approach used with human speech recognition that has been explored with bird sound is the use of Gaussian mixture models (GMM) (Kwan et al. 2004). In this approach, sounds are treated as a combination of normal distributions of sound elements, each with different densities or weights. Each test sound being classified has a different set of these mixtures and a classification is made based on the most likely combination estimated from sounds used to train the model. This method has been shown to be successful with a small data set of bird species, but as with the other approaches, it is susceptible to noise.

Perhaps the most common problem for automated identification of bird sound recorded in natural settings is noise. Not only can the presence of noise limit the ability for birdsong detection, it can also lead to misclassifications (Baker and Logue 2003). Far and away the most common method for dealing with noise is to limit the sound analysis to the frequency bands where the target sounds are found by using band-pass filters, as used by Farnsworth and Russell (2007) and Hüppop et al. (2006) to eliminate wind and ocean wave noise. Unfortunately, these methods can also eliminate many of the target sounds if they overlap the high noise part of the spectrum. Another technique is to use a sample of the background sound from a recording and subtract it out from the entire recording. This is most effective when the background noise is constant and not broad-band, and it has a limited utility with field recordings. A different approach is to apply a threshold filter at different frequency bands (Schrama et al. 2008). This has the effect of selecting sounds that are above the specified sound level threshold for analysis, but problems can arise with calls that vary in intensity. A different method to extract calls in a noise rich environment uses image processing of spectrograms (Brandes et al. 2006). In this approach, after a preliminary step of applying a threshold filter customized for each frequency band, a slight blur filter is applied to the spectrogram. This has the effect of washing out background noise, and applying a form of signal reconstruction to the calls, enabling a more consistent form of feature extraction. Though this method was tested mostly with cricket and frog calls from tropical forest recordings, it works equally well with detecting and classifying avian sounds that are constant in frequency such as those from tinamous (Tinamidae).

Methods to detect and classify avian sound in high noise environments have concentrated on extracting the peak frequency components from the songs. Tanttu et al. (2006) and Hüppop et al. (2006) were successful at detecting flight calls by using only the peak spectral components of calls that contained harmonics. Likewise, Chen and Maher (2006) use a method to track spectral peaks to classify bird sound recorded at airports. Spectral peak tracks are formed by segmenting the sound into short overlapping segments, measuring the peak frequency of that segment and matching it with the peak found in the adjacent sound segment. These tracks are then stored in a database and used as a template to classify the test sounds. This approach has shown to be successful with classifying some bird species, but often makes a classification only of general call type. In a different approach, a wide range of bird sounds can be detected and classified with a method that uses a noise reduction step through spectrogram image processing and adds to it a peak frequency contour feature measurement that is modelled with HMMs to classify frequency modulated whistles and complex sequences of them (Brandes 2008). Here, features are not extracted with cepstral coefficients, but instead with measurements of frequency, frequency bandwidth, and the change in these parameters from the previous time step. As with Trifa et al. (2008), this method has been shown to be effective at classifying groups such as antbirds; 
however, it has the advantage of using features that are less susceptible to background noise typical of tropical forests than cepstral coefficients, and is a method better suited to autonomous recordings. Additionally, Brandes (2008) employs hierarchical HMMs that find patterns at multiple levels of organization. Bottom-level HMMs detect calls, while higher level HMMs detect sequences of calls. This added pattern search ability allows for a much larger variety of call types to be searched for.

There is a variety of software available that can be used to automatically detect particular bird sounds, with proper training. None of them are comprehensive in their ability and they are all dependent on having moderate background noise and a good training set of bird sounds, particularly the software designed to work with human speech. We provide here a short list of free software; web addresses are provided with the reference list. For nocturnal flight calls of migrants, Farnsworth and Russell (2007) used software from Oldbird, Inc. Kogan and Margoliash (1998) and Trifa et al. (2008) used software titled the Hidden Markov Model Toolkit (HTK) from the Entropic Research Laboratory that is a software library written in C++. One software package particularly useful in developing avian sound recognition algorithms is the Extensible Bioacoustics Tool (XBAT) developed and distributed by the Bioacoustics Research Program at the Cornell Lab of Ornithology (Figueroa and Robbins 2008). This software runs as a toolbox within the MATLAB ${ }^{\circledR}$ mathematical programming environment. XBAT provides an environment designed to run standard detection algorithms, such as template matching, as well as an environment to test and develop custom designed bird sound recognition algorithms.

\section{Concluding thoughts}

The advent of this hardware and software is drawing bioacoustics into an ever increasingly important role in conservation. Conservation relies on documented sound recordings as a record of a species' acoustic signature as well as to document particular field records of observations that can be revisited. Likewise, automatic call recognition software relies on documented sound recordings, as they are only as good as the sound recordings used to train them. This places a particularly high importance on the role and necessity of sound libraries. Two extensive libraries are the Macaulay Library of Natural Sound at Lab of Ornithology at Cornell University and the library at the Borror Laboratory of Bioacoustics at Ohio State University. These libraries house many recordings from birds around the world, but they still lack many of the secondary calls which are not heard as often, and more effort is needed in collecting and donating recordings of bird calls that are more seldom heard (Kroodsma et al. 1996).

In many ways, the technology for automated recording of avian sound has arrived and the technology for automated analysis is burgeoning. This automation will be a big boon for science and conservation as it will greatly speed up and increase data collection from the field. This will provide more insight to avian ecology and conservation as well as allow for enhanced monitoring practices. For example, by having multiple recorders working simultaneously, automation can help to remove sampling biases during surveys due to temporal factors such as how chorus participants change throughout the morning. Also, by setting them to record before and after an observer arrives at a sampling location, they can help to detect whether or not standard field sampling techniques might be sensitive to observer disturbance on bird acoustic activity. The next steps for the hardware technology will be towards wireless networks that relay sound from remote locations back to central repositories. Additionally, more efficient solar cells will enable devices to have their own renewable power supply, allowing them to stay longer in the field, even when under the tree canopy.

Along with automated recorders that can last longer in the field and cost less, avian conservation needs software that can recognize all the avifauna calling in a region. This might require software that uses several algorithms simultaneously. Software that can effectively extract measurements from calls given in high background noise, as well as calls that overlap in time (even if not in frequency) is clearly required. Better yet, software that uses unsupervised or 
semi-supervised learning would greatly enhance the ability of bioacoustics to facilitate conservation, since often the slow point in the process is collecting sample recordings with which to train a classifier. Since many conservation efforts are undertaken in places with little scientific research history, the need to first have a database of sample sounds can create an impediment to automated bioacoustic monitoring. By using unsupervised learning algorithms, software would ideally be able to learn the different sounds in an area. The work would then be reduced to labelling the various types of sound to species afterwards. The technology used in bioacoustics is allowing conservation and science to advance rapidly in new ways and it looks poised to play an even bigger role in the near future.

\section{List of Web Addresses}

Borror Labroatory of Bioacoustics http://dmc.ohiolink.edu/ Cornell University's Bioacoustics Research Program http://www.birds.cornell.edu/brp/ Extensible Bioacoustic Tool (XBAT) http://xbat.org/home.html Hidden Markov Model Toolkit http://htk.eng.cam.ac.uk/

Macaulay Library of Natural Sound http://www.birds.cornell.edu/macaulaylibrary/

Oldbird, Inc. http://www.oldbird.org

River Forks Research Corp. http://rfrc1998.sasktelwebhosting.com/

Wildlife Acoustics, Inc. http://www.wildlifeacoustics.com/

\section{References}

Anderson, S. E., Dave, A. S. and Margoliash, D. (1996) Template-based automatic recognition of birdsong syllables from continuous recordings. J. Acoust. Soc. Am. Ioo: 1209-1219.

Angehr, G. R., Siegel, J., Acca, C., Christian, D. G. and Pequeno, T. (2002) An assessment and monitoring program for birds in the Lower Urubamba Region, Peru. Environ. Monit. Assess. 76: 69-87.

Baker, M. C. and Logue, D. M. (2003) Population differentiation in a complex bird sound: a comparison of three bioacoustical analysis procedures. Ethology 109: 223-242.

Becker, C. D. and Agreda, A. (2005) Bird community differences in mature and second growth Garua forest in Machalilla National Park, Ecuador. Ornitol. Neotrop. 16: 297-319.

Bibby, C. J., Burgess, N. D. and Hill, D. A. (1992) Bird census techniques. London: Academic Press.

Brandes, T. S. (2005) Acoustic monitoring protocol. Washington, DC: Conservation International. Tropical Ecology Assessment and Monitoring (TEAM) initiative set of biodiversity monitoring protocols. http:// www.teamnetwork.org (navigate to "Protocols").

Brandes, T. S. (2008) Feature vector selection and use with hidden Markov models to identify frequency-modulated bioacoustics signals amidst noise. IEEE Transactions on Audio, Speech, and Language Processing 16: (in press).

Brandes, T. S., Naskrecki, P. and Figueroa, H. K. (2006) Using image processing to detect and classify narrow-band cricket and frog calls. J. Acoust. Soc. Am. 120: 2950-2957.

Canterbury, G. E. and Blockstein, D. E. (1997) Local changes in a breeding bird community following forest disturbance. J. Field Ornithol. 68: 537-546.

Carpenter, L. (1978) A spectrum of nectareater communities. Am. Zool. 18: 809-819.

Chen, Z. and Maher, R. C. (2006) Semiautomatic classification of bird vocalizations using spectral peak tracks. J. Acoust. Soc. Am. 120: 2974-2984.

Collar, N. J., Crosby, M. J. and Stattersfield, A. J. (1994) Birds to watch 2: the world list of threatened birds. Cambridge, UK: Birdlife International.

Farnsworth, A., Gauthreaux, S. A. Jr. and Van Blaricom, D. (2004) A comparison of 
nocturnal call counts of migrating birds and reflectivity measurements on Doppler radar (WSR-88D). J. Avian Biol. 35: 365-369.

Farnsworth, A. and Russell, R. W. (2007) Monitoring flight calls of migrating birds from an oil platform in the northern Gulf of Mexico. J. Field Ornithol. 78: 279-289.

Feinsinger, P. and Colwell, R. K. (1978) Community organization among neotropical nectar-feeding birds. Am. Zool. 18: 779-795.

Figueroa, H. and Robbins, M. (2008) XBAT: an open-source extensible platform for bioacoustic research and monitoring. Pp. $143-155$ in K. H. Frommolt, R. Bardeli and M. Clausen, eds. Computational bioacoustics for assessing biodiversity. Proceedings of the International Expert meeting on IT-based detection of bioacoustical patterns, December 7 th until December 1oth, 2007 at the International Academy for Nature Conservation (INA), Isle of Vilm, Germany. BfN-Skripten vol. (234).

Fitzpatrick, J. W., Lammertink, M., Luneau Jr., M. D., Gallagher, T. W., Harrison, B. R., Sparling, G. M., Rosenberg, K. V., Rohrbaugh, R. W., Swarthout, E. C. H., Wrege, P. H., Swarthout, S. B., Dantzker, M. S., Charif, R. A., Barksdale, T. R., Remsen Jr., J. V., Simon, S. D. and Zollner, D. (2005) Ivory-billed woodpecker (Campephilus principalis) persists in continental North America. Science 308: 1460-1462.

Haselmayer, J. and Quinn, J. S. (2000) A comparison of point counts and sound recording as bird survey methods in Amazonian Southeast Peru. Condor 102: 887-893.

Hobson, K. A., Rempel, R. S., Greenwood, H., Turnbull, B. and Van Wilgenburg, S. L. (2002) Acoustic surveys of birds using electronic recordings: new potential from an omnidirectional microphone system. Wildl. Soc. Bull. 30: 709-720.

Holmes, R. T. (1990) Ecological and evolutionary impact of bird predation on forest insects: an overview. In M. L. Morrison, C. J. Ralph, J. Verner and J. R. Jehl, eds. Avian foraging: theory, methodologies and applications. Los Angeles: Cooper
Ornithological Society. Studies in Avian Biology no. 13.

Holmes, R. T., Schultz, J. C. and Nothnagle, P. (1979) Bird predation on forest insects: an exclosure experiment. Science 206: 462-463.

Howe, H. F. and Smallwood, J. (1982) Ecology of seed dispersal. Ann. Rev. Ecol. Syst. 13: $201-228$.

Hüppop, O., Dierschke, J., Exo, K. M., Fredrich, E. and Hill, R. (2006) Bird migration studies and potential collision risk with offshore wind turbines. Ibis 148 : 90-109.

Kogan, J. A. and Margoliash, D. (1998) Automated recognition of bird song elements from continuous recordings using dynamic time warping and hidden Markov models: A comparative study. J. Acoust. Soc. Am. 103: 2185-2196.

Kroodsma, D. E., Vielliard, J. M. E. and Stiles, F. G. (1996) Study of bird sounds in the Neotropics: urgency and opportunity. Pp. 269-281 in D. E. Kroodsma and E. H. Miller, eds. Ecology and evolution of acoustic communication in birds. Ithaca, NY: Cornell University Press.

Kwan, C., Mei, G., Zhao, X., Ren, Z., Xu, R., Standford, V., Rochet, C., Aube, J. and Ho, K. C. (2004) Bird classification algorithms: Theory and experimental results. IEEE Int. Conf. Acoust. Speech and Signal Processing (ICASSP'2004) V: 289-292.

Mackenzie, D. I., Nichols, J. D., Royle, J. A., Pollock, K. H., Bailey, L. L. and Hines, J. E. (2005) Occupancy estimation and modeling: Inferring patterns and dynamics of species occurrence. New York: Academic Press.

Parker, T. A. III. (1991) On the use of tape recorders in avifaunal surveys. Auk 108: 443-444.

Pounds, J. A., Fogden, M. P. L. and Campbell, J. H. (1999) Biological response to climate change on a tropical mountain. Nature 398: 611-615.

Proctor, M., Yeo, P. and Lack, A. (1996) The natural history of pollinators. Portland, OR: Timberline Press.

Ralph, C. J. and Scott, J. M., eds. (1981) Estimating numbers of terrestrial birds. Lawrence, KS: Cooper Ornithological Society. Studies in Avian Biology no. 6. 
Ralph, C. J., Guepel, G. R., Pyle, P., Martin, T. E. and DeSante, D. F. (1993) Handbook of field methods for monitoring landbirds. Albany, CA: Pacific Southwest Research Station, U. S. Forest Service.

Riede, K. (1993) Monitoring biodiversity: analysis of Amazonian rainforest sounds. Ambio 22: 546-548.

Riede, K. (1998) Acoustic monitoring of Orthoptera and its potential for conservation. J. Insect Conserv. 2: 217-223.

Rempel, R. S., Hobson, K. A., Holborn, G., Van Wilgenburg, S. L. and Elliott, J. (2005) Bioacoustic monitoring of forest songbirds: interpreter variability and effects of configuration and digital processing methods in the laboratory. J. Field Ornithol. 76: I-II.

Royle, J. A. and Nichols, J. D. (2003) Estimating abundance from repeated presence-absence data or point counts. Ecology 84: 777-790.

Schrama, T., Poot, M., Robb, M. and Slabbekoorn, H. (2008) Automated monitoring of avian flight calls during nocturnal migration. Pp. 131-134 in K. H. Frommolt, R. Bardeli and M. Clausen, eds. Computational bioacoustics for assessing biodiversity. Proceedings of the International Expert meeting on IT-based detection of bioacoustical patterns, December $7^{\text {th }}$ until December 1oth, 2007 at the International Academy for Nature Conservation (INA), Isle of Vilm, Germany. BfN-Skripten vol (234).

Tanttu, J. T., Turunen, J., Selin, A. and Ojanen, M. (2006) Automatic feature extraction and classification of crossbill (Loxia spp.) flight calls. Bioacoustics 15: 251-269.

Terry, A. M. R. and McGregor, P. K. (2002) Census and monitoring based on individually identifiable vocalizations: the role of neural networks. Anim. Conserv. 5: 103-111.

Trifa, V. M., Kirschel, A. N. G., Taylor, C. E. and Vallejo, E. E. (2008) Automated species recognition of antbirds in a Mexican rainforest using hidden Markov models. J. Acoust. Soc. Am. 123: 2424-2431.

Snow, D. W. (1971) Evolution aspects of fruit eating birds. Ibis 113: 194-202.

Snow, D. W. (1981) Tropical frugivorous birds and their food plants: a world survey. Biotropica 13: 1-4.

Verner, J. (1985) Assessment of counting techniques. Current Ornithol. 2: 247-302.

\section{T. SCOTT BRANDES*}

Conservation International, 2011 Crystal Drive, Suite 500, Arlington, VA, 22202, U.S.A. E-mail:tsbrandes@yahoo.com.

* Current address: Signal Innovations Group, Inc., 1009 Slater Road., Suite 200, Research Triangle Park, NC 27703, U.S.A. 